\title{
Pricing in Prosumer Aggregations using Reinforcement Learning
}

\author{
Utkarsha Agwan* \\ Electrical Engineering and Computer \\ Sciences, University of California, \\ Berkeley \\ United States of America \\ Tarang Srivastava \\ College of Letters and Sciences, \\ University of California, Berkeley \\ United States of America
}

\author{
Lucas Spangher* \\ Electrical Engineering and Computer \\ Sciences, University of California, \\ Berkeley \\ United States of America \\ Kameshwar Poolla \\ Electrical Engineering and Computer \\ Sciences, University of California, \\ Berkeley \\ United States of America
}

\author{
William Arnold \\ Electrical Engineering and Computer \\ Sciences, University of California, \\ Berkeley \\ United States of America
}

Costas J. Spanos

Electrical Engineering and Computer

Sciences, University of California,

Berkeley

United States of America

\begin{abstract}
Prosumers with generation and storage capabilities can supply energy back to the grid, or trade their surplus with other prosumers for their mutual benefit. A prosumer aggregation that facilitates such trades will price the energy being traded to achieve an objective such as profit maximization, social welfare, or market equilibrium. We propose the use of reinforcement learning to design a transactive controller to price energy in a prosumer aggregation. This has an advantage over other decentralized pricing mechanisms as it does not rely on iterative price settlement or load estimation by prosumers, and estimates the price in a day ahead manner. We present numerical case studies to evaluate our controller, and discuss extensions to implement this in real prosumer aggregations.
\end{abstract}

\section{CCS CONCEPTS}

- Computing methodologies $\rightarrow$ Reinforcement learning; • Hardware $\rightarrow$ Smart grid.

\section{INTRODUCTION}

Traditional consumers like buildings are increasingly investing in distributed energy resources such as solar panels and battery backups, and electrifying loads like vehicles. These resources can be used to supply the building's own demand, shave the peak load to reduce demand charges, or to increase resiliency in the face of grid failure or power shutoff and enable consumers to become prosumers, i.e. be electricity producers as well. However, such resources may remain underutilized, or be sized over-capacity to account for weather and load variability. Prosumers can profit from trading their surplus energy with other prosumers and improving resource utilization. Utilities have typically accommodated distributed energy resources

*Both authors contributed equally to this research.

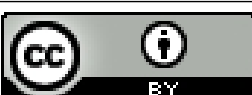

This work is licensed under a Creative Commons Attribution International 4.0 License. e-Energy '21, June 28-fuly 2, 2021, Virtual Event, Italy

(C) 2021 Copyright held by the owner/author(s).

ACM ISBN 978-1-4503-8333-2/21/06.

https://doi.org/10.1145/3447555.3464853

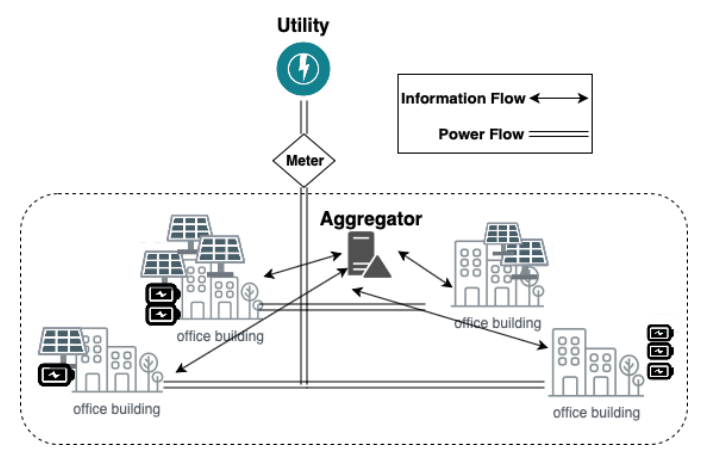

Figure 1: Social net metering in a prosumer aggregation

(DERs) through net-metering programs that compensate producers at the retail tariff. However, retail tariffs are much higher than wholesale energy market prices, and utilities have begun to phase out net metering programs in favor of direct market participation of DERs through aggregations. Prosumers can increase their profitability if they trade energy with other prosumers using social net-metering schemes implemented by utilities [7]. In these schemes, communities can share energy resources while presenting the net consumption to the utility as a single entity.

In this paper, we consider prosumer aggregations that facilitate trading between participants in the aggregation, and then balance the net load by purchasing from or selling to the utility. They can be formed with a variety of motives: private entities could manage aggregations for a fee or for a profit, and participants could form cooperative aggregations to maximize social welfare. Each aggregation has control the energy consumption and production of its participants, either through direct control or through some signal which can convey operational information to the participants. Realistically, prosumers will have independent cost minimization objectives, and will seek to optimize the operation of their resources for their own profit. Coordinating independent entities which are separately owned and managed is a difficult task, and transactive control is a strategy which uses the price of electricity to influence the operation of prosumers. For a prosumer, responding to a dayahead price is easier than estimating load/generation schedules 
required to participate in a market, or responding to real time prices. The aggregator can communicate time-of-use rates a day ahead to aggregation participants, who can then schedule their operation in response to energy prices similar to utility time-of-use rate plans. The aggregator has the task of designing prices that achieve the aggregation's objectives while dealing with an uncertain environment: first, the response of prosumers to energy prices is not known to the aggregator, and second, loads and generation are not perfectly predictable and have inherent occupancy and weather driven uncertainty.

Pricing resources in an uncertain environment is a task well suited for a data driven controller such as an RL algorithm, which motivates our work presented in this paper.

Contributions: We propose the use of Reinforcement Learning (RL) for pricing in local energy markets, and use it to develop a pricing mechanism that does not need explicit participant models or any operational information about prosumers. The proposed RL controller can replace iterative pricing methods commonly found in literature by learning to estimate market settlement and profit maximizing prices for the aggregator in a one-shot manner using historical and forecast data, and motivates further research in RL based methods for pricing.

\section{BACKGROUND}

In this section, we build background for the two main themes of this paper: prosumer aggregations and RL.

\subsection{Prosumer Aggregations}

Recent regulations have opened up multiple avenues for distributed energy resources (DERs) to participate in energy markets. However, wholesale markets have minimum participation sizes and may require DERs to construct demand and supply bids. Virtual microgrids and DER aggregations offer a pathway for prosumers to trade energy locally instead of participating in energy markets.

Researchers have worked on developing methods to control such aggregations. While microgrids have traditionally controlled distributed resources through a central authority dictating consumption/generation decisions, this can not be implemented in a situation where self-interested prosumers want to aggregate without ceding control of their operational decisions. [10] considers a microgrid central controller trying to shape the load curves of participants by employing participant differentiated real time pricing. [9] studies the problem of minimizing deviation from day ahead estimates through pricing, and [26] models a hierarchical optimization problem to solve the aggregation control problem. We model a similar hierarchical optimization in Section 3.

Aggregations commonly employ iterative pricing methods: [25] models prosumer trades as a Nash Bargaining problem, and solves it by decomposing it into two sequential problems which are solved iteratively using ADMM. This involves communicating price and energy consumption information back and forth between the aggregator and participants. Similarly, [13] develops a pricing model for a prosumer aggregation but settles on a price in an iterative manner. These methods have a couple of disadvantages: first, they require the participants to communicate back-and-forth with the aggregator which requires two-way communication infrastructure; second, prosumers are required to develop demand forecasts, which can unnecessarily raise the computational barrier for entry.

There is a need to devise methods to optimize the aggregator objective without relying on participant load/generation forecasts and without an iterative back and forth with prosumers, and this is the gap we try to address in this work.

\subsection{Reinforcement Learning}

$\mathrm{RL}$ is a type of agent-based machine learning where a complex system is controlled through actions that optimize the system in some manner [23]. The actions seek to optimize the expected sum of rewards for actions $\left(a_{t}\right)$ and states $\left(s_{t}\right)$ in a policy parameterized by $\theta$; i.e., $J(\theta)=\mathbb{E} \sum_{s_{t}, a_{t} \sim p_{\pi}}\left[r\left(s_{t}, a_{t}\right)\right]$. RL has been applied to a number of demand response situations in prosumer microgrids, but almost all the work centers on agents that directly schedule resources [24]. RL architectures can vary widely, for example [11] deploys a fuzzy Q-learning multi-agent that learns to coordinate appliances to increase reliability. In another illustrative example, [15] manages a battery directly using batch Q-learning. However, there are few works where the RL controller is a price setter in a market. In [14] RL has been used to estimate dynamic prices in a multi agent environment of demand response assets. To the best of our knowledge, RL has not been used to preemptively solve for equilibrium price in markets.

We propose the use of Soft Actor Critic, an RL architecture that tries to maximize the entropy of its actions to better explore the search space [6]. Actor-Critic architectures are composed of policy networks that suggest actions (actors) and value estimation networks that estimate the expected reward of the next state (critics), thereby guiding the actor network's learning [12]. How an agent prioritizes exploration vs. exploitation can be influenced by the inclusion of an entropy parameter $e$ which is valued by an entropy maximizing framework, i.e. $J(\theta)=\mathbb{E} \sum_{s_{t}, a_{t} \sim p_{\pi}}\left[r\left(s_{t}, a_{t}\right)+w_{e} e\right]$, weighting $e$ by some value $w_{e}$.

\section{METHODS}

We model the prosumer and aggregator behavior as solutions to optimization problems, and then introduce the RL controller that we use to estimate prices in a day ahead manner.

\subsection{Prosumer Model}

A prosumer typically has a combination of loads (flexible and inflexible), local generation and energy storage. We can denote the net energy consumption as $z^{(t)}=d^{(t)}-g^{(t)}+u^{(t)}$ where in any time period $t$, the prosumer has energy demand $d^{(t)}$, local generation $g^{(t)}$, and storage operation $u^{(t)}$. The prosumer purchases its net load at a time-of-use rate $\pi_{b}(t)$, and sells back any excess generation at $\pi_{s}(t)$. These prices are typically different [7], as utilities remove or disincentivize net-metering programs. The prosumer optimization problem (P-OPT) can be formulated as

$$
\begin{array}{rc}
\operatorname{P-OPT}\left(\boldsymbol{\pi}_{b}, \boldsymbol{\pi}_{s}\right): \min _{\mathbf{u}} & \sum_{t=1}^{T}\left[\pi_{b}^{(t)} z_{+}^{(t)}+\pi_{s}^{(t)} z_{-}^{(t)}+\pi_{b a t}\left|u^{(t)}\right|\right] \\
& =\boldsymbol{\pi}_{b}^{\top} \mathbf{z}_{+}+\boldsymbol{\pi}_{s}^{\top} \mathbf{z}_{-}+\pi_{b a t} \mathbf{1}_{T}^{\top}|\mathbf{u}| \\
\text { s.t. } & 0 \leq L \mathbf{u} \leq c
\end{array}
$$


where $\mathbf{u}$ represents the vector of battery charge/discharge over time with positive values denoting battery charging, $\boldsymbol{\pi}_{\boldsymbol{b}}, \boldsymbol{\pi}_{\boldsymbol{s}}$ represent the time vectors of buy and sell prices, and $\mathbf{z}_{+}, \mathbf{z}_{-}$represent the time vectors for net positive demand and net negative demand (net generation) respectively. The optimization objective (1a) incorporates the cost of procuring any net demand $z_{+}$at the buy price, the revenue from selling any net surplus energy generation $z_{-}$at the sell price, as well as the cost of battery degradation evaluated with $\pi_{b a t}$. The constraint (1c) encapsulates physical constraints on the state of charge for energy storage, charging speed constraints, and the one way battery efficiency $\eta$. More details can be found in [1].

\subsection{Aggregator Model}

Aggregators can be operated as central clearing houses where energy trades are balanced, and the net consumption is procured from the utility which acts as the outside option. All prosumers purchase their net energy needs from the aggregator at a price set by the aggregator. The aggregator is constrained in its choice of prices: if it is worse then the outside option (the utility), prosumers will have no incentive to trade with it. This constraint is encapsulated as $\pi_{s} \leq \lambda_{s}, \lambda_{b} \leq \pi_{b}$, where $\lambda_{s}, \lambda_{b}$ represent the aggregator-set sell and buy prices respectively. Aggregations can be formed with multiple objectives, and we explore two particular objectives: profit maximization and market balancing.

3.2.1 For-profit Aggregator. Aggregators can aim to maximize the profit they earn for acting as a trade facilitator, and in a situation with perfect information they would solve the following bilevel optimization problem to set prices:

$$
\begin{array}{cc}
\max _{\lambda_{b}, \lambda_{s}} & {\left[\lambda_{b}^{\top} \sum\left(\mathbf{z}_{+}^{*}\right)+\lambda_{s}^{\top} \sum\left(\mathbf{z}_{-}^{*}\right)\right]-\left[\boldsymbol{\pi}_{b}^{\top}\left(\sum \mathbf{z}^{*}\right)_{+}+\boldsymbol{\pi}_{s}^{\top}\left(\sum \mathbf{z}^{*}\right)_{-}\right]} \\
\text {s.t. } & \boldsymbol{\pi}_{s} \leq \boldsymbol{\lambda}_{s}, \lambda_{b} \leq \boldsymbol{\pi}_{b} \\
& \mathbf{z}^{*}=\mathbf{d}-\mathbf{g}+\mathbf{u}^{*} ; \mathbf{u}^{*}=\underset{\mathbf{u}}{\arg \min \operatorname{P-OPT}\left(\lambda_{b}, \lambda_{s}\right)}
\end{array}
$$

where the objective in Eq. 2a represents the net profit for the aggregator, i.e. the revenue from sales to the prosumers minus the cost of procuring the net energy demand from the utility. $\sum\left(\mathbf{z}_{+}^{*}\right), \Sigma\left(\mathbf{z}_{-}^{*}\right)$ are the sum of each prosumer's demand and generation taken separately, while $\left(\sum \mathbf{z}^{*}\right)_{+},\left(\sum \mathbf{z}^{*}\right)_{-}$are the net demand and generation of the aggregation once all internal trades have been balanced.

3.2.2 Market Solving Aggregator. The aggregation can be set up to operate a local energy market where prosumers negotiate with each other and eventually trade at the market equilibrium price. Instead of requiring prosumers to develop demand and supply bids and engage in negotiations, the aggregator can set prices that reflect the market equilibrium in a day ahead manner. Such an aggregator will aim to reduce the difference between day ahead estimated price and market settlement price, which is equivalent to ensuring a net zero profit/loss situation for the aggregator. This problem can be formulated as

$$
\begin{array}{cc}
\min _{\lambda_{b}, \lambda_{s}} & \|\left[\lambda_{b}^{\top} \Sigma\left(\mathbf{z}_{+}^{*}\right)+\lambda_{s}^{\top} \Sigma\left(\mathbf{z}_{-}^{*}\right)\right]-\left[\pi_{b}^{\top}\left(\sum \mathbf{z}^{*}\right)_{+}+\pi_{s}^{\top}\left(\sum \mathbf{z}^{*}\right)_{-}\right] \mid(3 \mathrm{a}) \\
\text { s.t. } & \text { Eq. 2b, Eq. 2c }
\end{array}
$$

Note that the objective (Eq. 3a) is the absolute value of the objective for the for-profit aggregator (Eq. 2a).

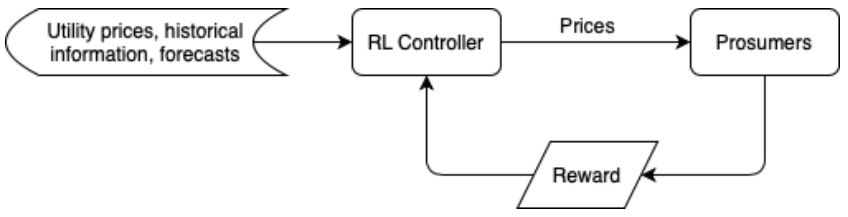

Figure 2: Reinforcement Learning control flow

\subsection{Transactive Control using RL}

The problems modeled in Section 3.2 are hierarchical optimization problems, and do not have a closed-form solution without some form of information sharing between the aggregator and prosumers. As discussed in other papers which use ADMM and iterative pricing methods (Section 2), decentralized pricing methods require iterations to converge to a solution. We develop an RL controller that relies on a day-ahead price set using historical price information and generation forecasts. The transactive controller does not iterate over prices, and instead learns to estimate future prices in a one-shot manner. Our search space of possible prices is simple enough to be covered by an entropy maximizing agent, and we employ a Soft Actor-Critic (SAC) architecture to do so. The reward for the for-profit aggregator is computed as the objective expressed in Eq. 2a, and the reward for the market solving aggregator is computed by the objective in Eq. 3a. We simulate the behavior of our controller under uncertain generation forecasts and compare it to baseline iterative pricing algorithms in Section 4.

\section{RESULTS}

We will now describe our results for each of the two objectives: market solving and profit maximization, prefaced by an explanation of our data, architecture and training process. We model this problem after an environment to simulate demand response in office buildings [21]. Each step in the environment is a day where the RL controller broadcasts prices to the prosumers, who modify their energy storage and consumption to minimize their costs, and the controller uses their consumption data to calculate its reward.

Implementation. We use the stable-baselines fork of OpenAI baselines [8], and our other implementation choices are detailed in our Github repository [20]. The final run presented here was distributed across 24 CPUs for 12 hours each. The Q-factor loss shown in Fig. 5 is one of many metrics that represents the neural network's training. The market solving controller's reward (Eq. 3a) is always negative. For the simulations presented in this paper, the utility pricing is obtained from [17] and the prosumers considered are commercial office buildings modeled using load data taken from [16] with additional details presented in [1].

\subsection{Marginal Benefit of Aggregating}

We compare the system costs in the presence and absence of a profit-maximizing aggregator for two different levels of prosumer solar generation and battery capacity. As can be see in Fig. 3, the $\mathrm{RL}$ controller reduces the system costs and provides value which can be distributed among the aggregator and prosumers. 

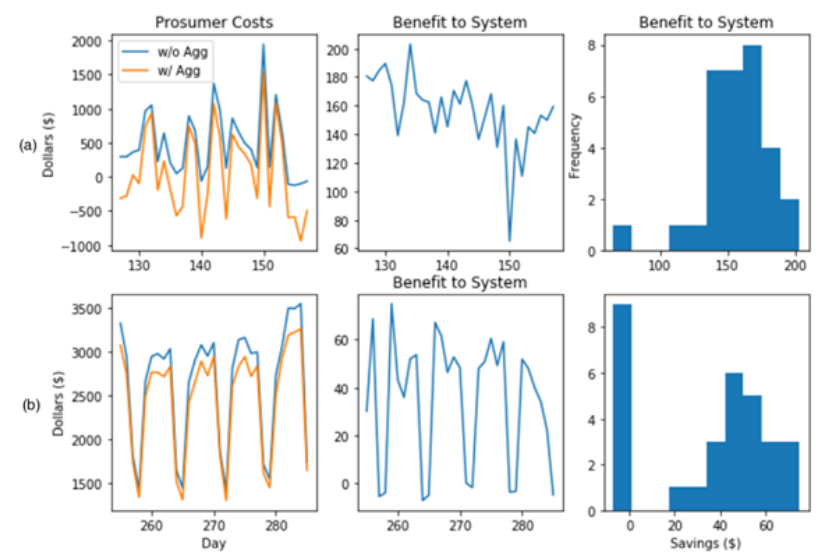

Figure 3: Comparing system costs, i.e. sum of aggregator and prosumer costs with and without a profit maximizing RL controller for two resource levels: a) Medium, and b) Small

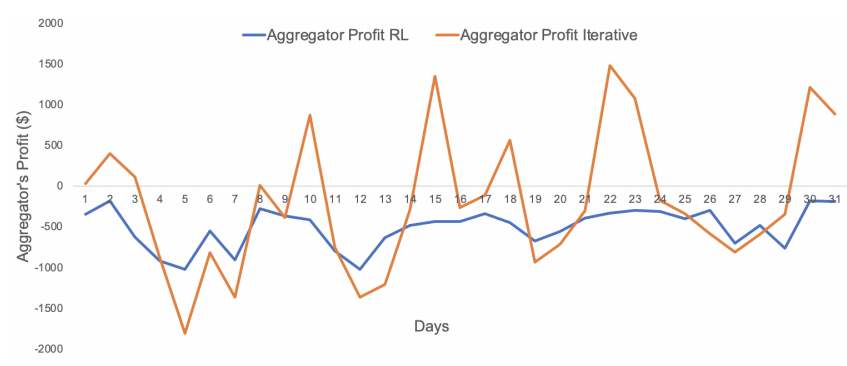

Figure 4: Comparing aggregator profit for market solving prices with iterative pricing and $\mathrm{RL}$ controller
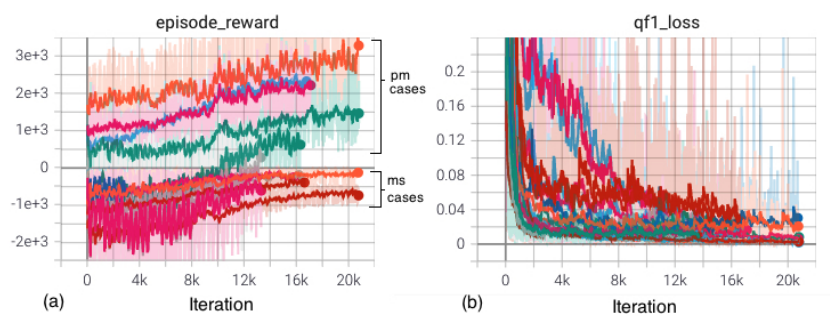

Figure 5: Training curves for reward and Q-factor loss. The concave shape indicates an approach to convergence

\subsection{Comparison with Iterative Pricing}

We compare the market solving RL controller with an iterative pricing scheme that aims to achieve market equilibrium through back and forth negotiations with prosumers [1]. In this case, the objective is to drive the aggregator profit close to zero. The RL controller has a smoother profit on average, i.e it exhibits lower deviations than the iterative pricing algorithm as can be see in Fig. 4. We believe that expanding training and shaping the observation space could potentially improve the performance of the RL controller.

\section{DISCUSSION}

RL controllers are particularly well suited for dynamic data driven environments with unknown, complex, and changing system models, e.g. with changing prosumer resources. The novelty of our proposition is in using RL to preemptively price energy in local markets, but is accompanied by many challenges that will need to be addressed for practical applicability.

Safety: An RL controller might generate prices that result in infeasible operation. We tackle this by using utility-set prices to enforce limits on the aggregator prices $\lambda_{s}, \lambda_{b} \in\left[\boldsymbol{\pi}_{s}, \boldsymbol{\pi}_{b}\right]$. An additional safety check would involve validating these prices through a single back-and-forth communication with prosumers to ensure that their operation does not exceed system limits. Probabilistic guarantees for RL controllers can be used [2]. Additionally, supervised RL can help guarantee safety at the outset [19].

Efficiency: While the large number of training iterations represent a greater computational burden than iterative pricing methods, the RL controller will reduce the computational burden on each individual prosumer by eliminating the need to construct forecasts or demand/supply bids. Further, each prosumer's computations occur in parallel and adding new prosumers to the aggregation does not increase the problem complexity.

Robustness: Adversarial training is a useful method to construct robust RL controllers [18], and can be extended to our setup.

Optimality: Research has been done in bounding the sub-optimality of RL policies using policy certificates [3], and research in this area can help provide guarantees for our RL controller as well.

Practical Implementation: The training iterations needed are a barrier to real-life use of an RL controller in a prosumer aggregation. However, there are numerous enhancements we propose to address this. First, meta-learning: a large part of the training can take place in a simulation environment which can use a rules-based heuristic as a starting point, and use exogenous parameters to create a distribution of unique systems to train on (i.e. domain randomization.) A technique like Model Agnostic Meta-Learning can train in the different simulations to approximate a starting distribution for policy network weight initializations [4]. However, the accuracy of the simulation model will determine how effective the RL controller is when it transitions to an actual aggregation. Second, planning: a Dyna-like auxiliary model, either generative (i.e. GANs) or predictive (i.e. regression or neural nets), could train with the agent and help augment data [22]. Third, offline learning can incorporate data from other microgrids using techniques in the causal methods literature to help adequately perform the data fusion necessary[5]. However, validating these ideas will require more work.

In real life, either formulation of the aggregator could be implemented: a profit-seeking entity could operate the controller, or a prosumer cooperative could aim to facilitate trades between members. In this paper, we model the utility as the outside alternative. As prosumer aggregations grow larger, they may participate in wholesale energy markets instead of purchasing energy from utilities, and will have to adapt to variable energy market prices as well as having to generate estimates of their own consumption and production. RL algorithms have been previously used to optimize market participation, and these would be complementary to the model we present here. 


\section{REFERENCES}

[1] Utkarsha Agwan. 2020. Optimal Prosumer Aggregations: Design and Modeling. (2020).

[2] Edoardo Bacci and David Parker. 2020. Probabilistic guarantees for safe deep reinforcement learning. In International Conference on Formal Modeling and Analysis of Timed Systems. Springer, 231-248.

[3] Christoph Dann, Lihong Li, Wei Wei, and Emma Brunskill. 2019. Policy certificates: Towards accountable reinforcement learning. In International Conference on Machine Learning. PMLR, 1507-1516.

[4] Chelsea Finn, Pieter Abbeel, and Sergey Levine. 2017. Model-Agnostic MetaLearning for Fast Adaptation of Deep Networks. CoRR abs/1703.03400 (2017). arXiv:1703.03400 http://arxiv.org/abs/1703.03400

[5] Andrew Forney and Elias Bareinboim. 2019. Counterfactual Randomization: Rescuing Experimental Studies from Obscured Confounding. Proceedings of the AAAI Conference on Artificial Intelligence 33, 01 (Jul. 2019), 2454-2461. https //doi.org/10.1609/aaai.v33i01.33012454

[6] Tuomas Haarnoja, Aurick Zhou, Pieter Abbeel, and Sergey Levine. 2018. Soft actor-critic: Off-policy maximum entropy deep reinforcement learning with a stochastic actor. In International Conference on Machine Learning. PMLR, 18611870.

[7] Rodrigo Henriquez-Auba, Patricia Pauli, Dileep Kalathil, Duncan S Callaway, and Kameshwar Poolla. 2018. The Sharing Economy for Residential Solar Generation. In 2018 IEEE Conference on Decision and Control (CDC). IEEE, 7322-7329.

[8] Ashley Hill, Antonin Raffin Maximilian Ernestus, Adam Gleave, Anssi Kanervisto, Rene Traore, Prafulla Dhariwal, Christopher Hesse, Oleg Klimov, Alex Nichol, Matthias Plappert, Alec Radford, John Schulman, Szymon Sidor, and Yuhuai Wu. 2018. Stable Baselines. https://github.com/hill-a/stable-baselines.

[9] Liyan Jia, Qing Zhao, and Lang Tong. 2013. Retail pricing for stochastic demand with unknown parameters: An online machine learning approach. In 2013 51st Annual Allerton Conference on Communication, Control, and Computing (Allerton) IEEE, 1353-1358

[10] Seung-Jun Kim and Geogios B Giannakis. 2016. An online convex optimization approach to real-time energy pricing for demand response. IEEE Transactions on Smart Grid 8, 6 (2016), 2784-2793.

[11] P. Kofinas, A.I. Dounis, and G.A. Vouros. 2018. Fuzzy Q-Learning for multi-agen decentralized energy management in microgrids. Applied Energy 219 (2018), 53-67. https://doi.org/10.1016/j.apenergy.2018.03.017

[12] Vijay R Konda and John N Tsitsiklis. 2000. Actor-critic algorithms. In Advances in neural information processing systems. Citeseer, 1008-1014.

[13] Nian Liu, Xinghuo Yu, Cheng Wang, Chaojie Li, Li Ma, and Jinyong Lei. 2017 Energy-sharing model with price-based demand response for microgrids of peer to-peer prosumers. IEEE Transactions on Power Systems 32, 5 (2017), 3569-3583.

[14] Renzhi Lu, Seung Ho Hong, and Xiongfeng Zhang. 2018. A dynamic pricing demand response algorithm for smart grid: reinforcement learning approach. Applied Energy 220 (2018), 220-230.

[15] Brida V. Mbuwir, Frederik Ruelens, Fred Spiessens, and Geert Deconinck. 2017. Battery Energy Management in a Microgrid Using Batch Reinforcement Learning. Energies 10, 11 (2017). https://www.mdpi.com/1996-1073/10/11/1846

[16] Clayton Miller and Forrest Meggers. 2017. The Building Data Genome Project: An open, public data set from non-residential building electrical meters. Energy Procedia 122 (2017), 439-444.

[17] OpenEI. 2017. Time of Use pricing. https://openei.org/apps/USURDB/rate/view/ 5cbf78b25457a34e40671081\#3 Energy

[18] Anay Pattanaik, Zhenyi Tang, Shuijing Liu, Gautham Bommannan, and Girish Chowdhary. 2017. Robust deep reinforcement learning with adversarial attacks. arXiv preprint arXiv:1712.03632 (2017)

[19] M. Rosenstein and A. Barto. 2002. Supervised Learning Combined with an ActorCritic Architecture TITLE2: Technical Report. USA.

[20] Lucas Spangher, Utkarsha Agwan, William Arnold, and Tarang Srivastava. 2021 . https://github.com/utkarshapets/microgrid-RL

[21] Lucas Spangher, Akash Gokul, Joseph Palakapilly, Utkarsha Agwan, Manan Khattar, Wann-Jiun Ma, and Costas Spanos. 2020. OfficeLearn: An OpenAI Gym Environment for Reinforcement Learning on Occupant-Level Building's Energy Demand Response. In Tackling Climate Change with Artificial Intelligence Workshop at NeurIPS, 2020

[22] Richard S. Sutton. 1991. Dyna, an Integrated Architecture for Learning, Planning, and Reacting. SIGART Bull. 2, 4 (July 1991), 160-163. https://doi.org/10.1145/ 122344.122377

[23] Richard S Sutton and Andrew G Barto. 2018. Reinforcement learning: An introduction. MIT press.

[24] José R Vázquez-Canteli and Zoltan Nagy. 2019. Reinforcement learning for demand response: A review of algorithms and modeling techniques. Applied energy 235 (2019), 1072-1089.

[25] Hao Wang and Jianwei Huang. 2016. Incentivizing energy trading for interconnected microgrids. IEEE Transactions on Smart Grid 9, 4 (2016), 2647-2657.

[26] Yu Wang, Shiwen Mao, and R Mark Nelms. 2015. On hierarchical power schedul ing for the macrogrid and cooperative microgrids. IEEE Transactions on Industria
Informatics 11, 6 (2015), 1574-1584.

\section{ACKNOWLEDGMENTS}

This work is supported by the Republic of Singapore's National Research Foundation through a grant to the Berkeley Education Alliance for Research in Singapore (BEARS) for the Singapore-Berkeley Building Efficiency and Sustainability in the Tropics (SinBerBEST) program 